Revue d'histoire de l'enfance « irrégulière »

Le Temps de l'histoire

Hors-série | 2007

Pages d'histoire, la protection judiciaire des mineurs, $\mathrm{XIX} \mathrm{X}^{\mathrm{e}} \mathrm{XX} \mathrm{X}^{\mathrm{e}}$ siècles

\title{
Histoire de mots
}

Jacques Bourquin, Begnino Cacérès et Vincent Peyre

\section{(2) OpenEdition \\ 1 Journals}

Édition électronique

URL : http://journals.openedition.org/rhei/3027

DOI : $10.4000 /$ rhei.3027

ISBN : 978-2-7535-1647-2

ISSN : $1777-540 \mathrm{X}$

Éditeur

Presses universitaires de Rennes

Édition imprimée

Date de publication : 1 juin 2007

Pagination : $321-326$

ISSN : $1287-2431$

Référence électronique

Jacques Bourquin, Begnino Cacérès et Vincent Peyre, " Histoire de mots », Revue d'histoire de l'enfance «irrégulière » [En ligne], Hors-série | 2007, mis en ligne le 01 février 2010, consulté le 30 avril 2019. URL : http://journals.openedition.org/rhei/3027 ; DOI : 10.4000/rhei.3027 


\section{Histoire de mots ${ }^{(*)}$}

Pervers, malheureux ou déficients, à corriger, à régénérer ou à réadapter sous la férule de surveillants ou grâce aux soins éclairés d'éducateurs et de psychologues, les mineurs de justice suscitent à la fois crainte et compassion. Ces représentations contradictoires persistent tout au long d'un siècle et demi et plus d'histoire, provoquant un débat sans cesse renouvelé, jusqu'à aujourd'hui, sur les parts respectives de la répression (et de la pénitence) ou de l'éducation (et de la cure médico-psychologique) dans un traitement dont la nécessité n'est guère questionnée. C'est pourquoi, même si certains termes ont vieilli et si en particulier le vocabulaire s'est laïcisé, on a bien de la peine à découvrir des ruptures franches. On note bien plutôt des résurgences. On peut se demander s'il y a très loin de la régénération à la restructuration de la personnalité, ou des colonies agricoles (1835) aux chantiers de jeunesse (1986). Est-ce à dire que rien ne change ? Certainement pas. Les " bagnes d'enfants" ont bel et bien disparu, comme les « miséricordes » où les filles étaient enfermées jusqu’à 21 ans. C'est dire que rien ici n'est acquis à jamais, qu'il n'y a pas un mouvement uniforme et rectiligne de "progrès "... et que les meilleures intentions méritent d'être interrogées à la lumière de l'histoire.

\section{Petit glossaire de l'éducation surveillée et des mineurs de justice Quelles finalités et quels objectifs ?}

Régénération. "Les mineurs non discernants sont acquittés remis à leur famille ou à l'Administration pénitentiaire en vue d'être placés en maison corrective pour y être éduqués et régénérés. » (Code pénal, 1810)

"Les jeunes délinquants seront régénérés par la vocation agricole. » (Charles Lucas, fondateur de la colonie agricole du Val-Yêvre, 1843)
(*) Texte rédigé par Jacques Bourquin, Begnino Cacérès et Vincent Peyre, publié dans la revue Pour, Privat, 1987.

Histoire de mots / p. 321 à 326 
Rédemption. «Des Pénitentes aux Madeleines (les jeunes repenties) s'est établi un circuit puissant de la rédemption. » (Mère Marie Euphrasie Pelletier, fondatrice du Bon Pasteur, 1850)

Correction. "Prévoir une éducation spéciale en maison de correction. » (Loi de 1791 sur les mineurs délinquants, les maisons n’apparaîtront que vers 1830)

Moralisation et Amendement. " Les tribunaux de la Seine convaincus de l'efficacité de nos moyens combinés de moralisation se montrent plus disposés qu'auparavant à ordonner, pour un temps plus ou moins long, l'emprisonnement à titre de correction des délinquants que leur âge rend plus susceptibles d'amendement. » (Béranger de la Drôme, fondateur de la société de patronage des jeunes libres, 1840)

"Votre fils nous a été confié pour une certaine durée, c'est qu'il a été jugé que son amendement pourrait être long. » (IPES Aniane, 1960, note aux parents) Ces trois termes correction, moralisation, amendement sont indissociables jusqu'à la veille de la seconde guerre mondiale. Le terme « correction » subsiste dans l'opinion publique, celui d'" amendement » disparaîtra du langage de l'Éducation surveillée vers 1960.

Réformation. "L'œuvre de réformation des filles devient en quelque sorte impossible lorsqu'elles ont été souillées par la prostitution. " (Édouard Ducpetiaux, 1837)

«Est-ce sensé de vouloir réformer 500 ou 600 enfants remis dans les mêmes murs?" (Henri Joly, L'enfance coupable, 1904)

Relèvement. "Relever les jeunes coupables et leur donner la force de se laisser ramener au bien, voilà certes une grande tâche. » (Henri Joly, L'enfance coupable, 1904)

" Nous invitons la famille à collaborer au relèvement moral de son enfant. » (IPES Aniane, 1960, notice envoyée aux parents)

Redressement. «Les partisans des procédés miel et sucre prétendent que la persuasion doit suffire pour contenir et redresser les jeunes malfaiteurs... » (Un instituteur chef, colonie Eysses, 1899)

« Instituteurs et institutrices sont chargés du redressement moral. » (Règlement des maisons d'éducation surveillée, 1935)

"L'union a contribué à la construction et à l'amélioration des nouveaux régimes de redressement des enfants "indisciplinés", "dévoyés", "vagabonds" 
ou "coupables". "(Union des sociétés de patronage)

Le terme de redressement disparaîtra progressivement pour faire place à celui de rééducation.

Rééducation. "La rééducation cède la place à la répression. " (Alexis Danan, journaliste, 1937)

"Les méthodes de rééducation. " (Titre d'un chapitre du Plan de réforme de l'Éducation surveillée, 1946)

La rééducation est le terme consacré par l'ordonnance du 2 février 1945 relative à l'enfance délinquante.

Normalisation. "La normalisation présente un intérêt évident en milieu ouvert du fait que l'enfant subit la pression du milieu réel. » (Paul Lutz, inspecteur de l'Éducation surveillée, 1959)

Reconditionnement. "Le reconditionnement vise à deux fins : faire acquérir à l'enfant des réflexes conditionnés à travers des processus d'imprégnation et d'identification et à être pénétré par l'ambiance d'un groupe et d'un style de vie. " (Jean Chazal, magistrat, 1953)

Reclassement. "L'action de l'internat a pour but de préparer le reclassement social. » (Paul Lutz, 1950)

Réadaptation. "La finalité de la rééducation, c'est la réadaptation sociale du mineur. » (Jean-Louis Costa, 1951, premier directeur de l'Éducation surveillée en 1945)

Réintégration. "À l'issue du retour en IPES, la réintégration sociale du jeune doit présenter toutes les garanties favorables. » (IPES Aniane, 1960)

Restructuration de la personnalité. "La rééducation entreprise, la restructuration de la personnalité déborde largement le reconditionnement. » (Henri Michard, directeur du Centre d'études, de formation et recherches de l'Éducation surveillée, Vaucresson, 1965)

Réinsertion. "En internat, la réinsertion sociale se fait progressivement, elle est préparée par les permissions et la collaboration des services de milieu ouvert. " (Note de présentation de l'Éducation surveillée, 1969)

Insertion et Autonomisation. "Il doit y avoir le souci constant d'aboutir le plus rapidement possible à l'autonomisation du mineur. " (Orientation de l'Éducation surveillée, 1979)

« L'Éducation surveillée doit veiller à combler les lacunes et les handicaps des 
jeunes qui lui sont confiés dans le souci de faciliter leur autonomie et leur insertion sociale. " (Circulaire Éducation surveillée, 1983)

Les termes « insertion » et « autonomisation » sont fréquemment employés depuis les années 1975.

Réparation. «La rééducation du jeune s'apparente à la réparation d'un bâtiment vétuste qu'il faut transformer, remettre à neuf, intérieurement et extérieurement. » (François Dhalenne, directeur de l'IPES de Saint-Hilaire, 1947) " Dans le cadre de l'ordonnance de 1945, se sont développées empiriquement, sous l'impulsion des éducateurs, des procédures de mise en œuvre du principe de réparation. Tant au plan matériel que juridique et symbolique. » (François Colcombet, 1984, directeur de l'Éducation surveillée)

Le terme est utilisé dans les années 1940-1950. Il réapparaît dans les années 1980, chargé d'un autre sens, au moment où l'Éducation surveillée tente de promouvoir une politique de diminution des incarcérations et de prise en compte de l'intérêt des victimes.

Jeunes : les mots pour « les » dire

Enfance coupable

Enfance déficiente

Enfance délinquante

Enfants de justice

Enfance victime
Enfance irrégulière

Jeunesse délinquante

Enfance criminelle

Enfance inadaptée

Enfance malheureuse

On évoque aussi les « insoumis ", les « irrécupérables », les « inamendables », les «vicieux », les " dévoyés ». Beaucoup plus récemment, on entend parler des « incasables "

\section{La Justice les appelle :}

les colons, 1835

les pupilles, 1927

les élèves, dès les réformes de 1937

\section{Les éducateurs évoquent :}

les gosses, à partir de 1938

(employé au XIXème siècle)

les tapirs (argot d'internat), 1945

les jeunes (apparition du milieu ouvert), 1960

les ados (foyers)

les mineurs (terme générique) 
Classification nosologique utilisée... dans les colonies pénitentiaires, 1901 Intelligents

Vicieux

Indifférents

Arriérés

Anormaux

Dégénérés dans les centres d'observation, 1945-1955

Apathiques

Hyperémotifs

Instables

Cyclothymiques

Impulsifs

Paranö̈aques

Mythomanes

Schizoïdes

Pervers

Personnels d'enseignement professionnel

Maîtres-ouvriers, 1900

Sous-chefs et chefs d'atelier, 1935

Instructeurs, 1938

Instructeurs techniques, 1945

Professeurs techniques adjoints, 1966

Professeurs techniques d'enseignement professionnel, 1974

\section{Les institutions pour mineurs}

de la Pénitentiaire à l'Éducation surveillée

Maison d'amélioration (projet Mirabeau, 1790)

Maison de correction (loi 1791)

Prison d'amendement (loi 1814)

Maison d'éducation correctionnelle (1836)

Colonies agricoles (à partir de 1835)

Colonies industrielles (à partir de 1860)

Colonies pénitentiaires (1850)

Colonies correctionnelles (1850)

École de préservation (filles) (1900) 
École de réforme (moins de 13 ans) (1900)

Internat approprié (moins de 13 ans) (1912)

Maison d'éducation surveillée (1927)

Institution publique d'éducation surveillée (1940).

On les appelle souvent après 1945 :

internats publics d'éducation surveillée ou bien internats

professionnels d'éducation surveillée

Centre d'observation public d'éducation surveillée (1945)

Institution d'éducation corrective de l'Éducation surveillée

(1945-1951)

Institution spéciale d'éducation surveillée

(pour les mineurs les plus difficiles, 1952-1958)

Services d'observation de l'Éducation surveillée dans

les quartiers de mineurs (1958-1979)

Foyers de semi-liberté (à partir de 1955)

Foyers d'action éducative (à partir de 1965)

Institution spéciale d'éducation surveillée

(sans rapport avec les précédentes du même nom.

C'est la forme d'institution qui regroupe à partir de

1973 : hébergement, milieu ouvert, formation)

Le secteur des Bons Pasteurs pour les filles

Termes employés dans ce secteur au XIXème siècle et souvent jusqu'en 1945

$\begin{array}{lll}\text { les institutions } & \text { les jeunes filles } & \text { les finalités } \\ \text { Asiles } & \text { Pénitentes } & \text { Pénitence } \\ \text { Solitudes } & \text { Repentantes } & \text { Rachat } \\ \text { Miséricordes } & \text { Madeleines } & \text { Rédemption } \\ \text { Providences } & & \\ \text { Compassion } & & \\ \text { Refuges (dès le } & & \\ \quad \text { XVIIème siècle) } & & \end{array}$

